\title{
The Identification and Recognition Based on Point for Blood Vessel of Ocular Fundus
}

\author{
Zhiwen $\mathrm{Xu}^{1}$, Xiaoxin $\mathrm{Guo}^{1}$, Xiaoying $\mathrm{Hu}^{2}, \mathrm{Xu} \mathrm{Chen}^{1}$, and Zhengxuan Wang ${ }^{1}$ \\ 1 Open Symbol Computation and Knowledge Engineering Laboratory of State \\ Education Department, College of Computer Science and Technology \\ 2 The First Clinical Hospital, \\ Jilin University, Changchun City, 130012, Jilin Province, China \\ xuzhiwen@public.cc.jl.cn
}

\begin{abstract}
Today, iris recognition, fingerprint recognition, face recognition, voice recognition and other biometric technology are experiencing rapid development. This paper addresses a new biometric technologythe identification and recognition based on point of blood vessel skeleton for ocular fundus. The image for green gray scale of ocular fundus is utilized. The cross point of skeleton shape of blood vessel for ocular fundus using contrast-limited adaptive histogram equalization is extracted at first. After filtering treatment and extracting shape, shape curve of blood vessels is obtained. The cross point of shape for curve matching is later carried out by means of cross point matching. The recognition based on shape for blood vessel of ocular fundus has been demonstrated in this paper to possess high Identification and recognition rate, low rejection recognition rate as well as good universality, exclusiveness and stability. With more and more progress made in extracting technology, the recognition for blood vessel of optic fundus is to become an effective biometric technology.
\end{abstract}

\section{Related Work}

The shape of blood vessel for ocular fundus is important indicator to diagnose such diseases as hypertension, vascular sclerosis, coronary artery sclerosis and diabetes. The certain achievements have been fulfilled in image processing of blood vessel of ocular fundus. Considering that zero-crossing can neither always correspond to real edge nor always signal edge position precisely, F. Ulupinar[1] makes relevant revision. Canny[2] raises optimal operator suitable for any randomly shaped edge extraction. However, due to utilization of Gaussian filter, it still remains imprecise occasionally. Though arithmetic operator of morphological gradient [3] is easy and quick, it's confined to image with the noise of pepper and salt. The relax method is used to extract linear blood vessels. Since arteries and veins cross over each other, blood vessels have to be segmented into several pieces. Hueckel uses arithmetic operator to apply fitting method of blood vessel to extract edge[4]. It is insensitive to noise and effective in region of intense texture, but it demands tremendous calculation. Taking into consideration the inherent features of blood vessel [5], being that 
blood vessel is of linear shape and gradient direction on the left threshold is just opposite to that on the right threshold, Tascini G. puts forward the method of search edge direction [6]. The edge of blood vessel in the second period of hypertension is rather blur and very low contrast so that it can't be traced. In order to improve that, Rangayyan R. M. et al suggests reinforcing linear feature of blood vessel at the frequency field. But the result isn't good due to exudation of ocular fundus. Chauduri S. et al. employs the Gaussian models of twelve different directions to filter fundus blood vessel [7]. Then, those methods aren't nice at enhancing the fundus image of blood vessel for hypertension patients. Otherwise, utilization of Gaussian madel of fixed size makes it fail in dealing with blood vessels with salient variation in diameter and shape highly curved. Substitution of Gaussian models of different sizes is sure to cause excessive computation. The literatures $[8,9]$ make a study on matching for blood vessel of ocular fundus. The literatures $[10,11]$ make a study of algorithm of fingerprint based on point pattern. Based on the researches mentioned above, together with the blood vessel of Ocular Fundus for biometric features, this paper addressed the recognition for blood vessel of Ocular Fundus, using contrast-limited adaptive histogram equalization by employing gradient vector curve to analyze the features of blood vessel of ocular fundus.

\section{Feature to Extract Blood Vessel of Ocular Fundus}

The diameter of capillary vessels for ocular fundus may vary with passage of time and various diseases, then their direction for distribution remains the same for long time. This encourages vector curve that analyzes distribution of blood vessel orientation to be utilized as biometric features for blood vessel of ocular fundus. The concrete steps extracts feature as the following:

(1) Enhance contrast of gray scale image and utilize contrast limited adaptive histogram equalization. At first, deal with subregions in the image and then converge adjacent small regions with method of bilinear interpolation in order to get rid of artificial edges. Fig.1 is fundus image of gray scale; Fig.2 is gray scale enhanced image; Fig. 3 is inversion to enhance result of gray scale.

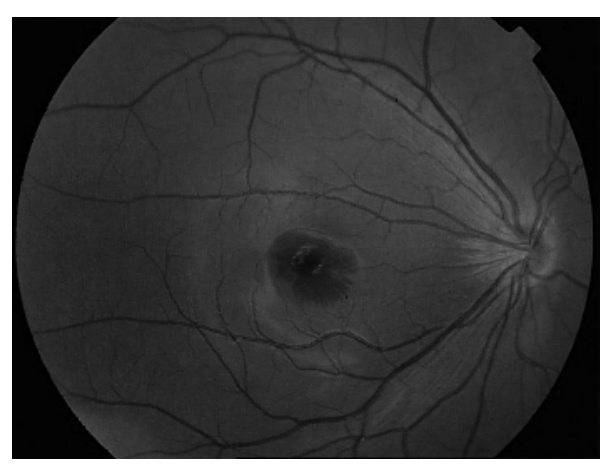

Fig. 1. Fundus image of gray scale

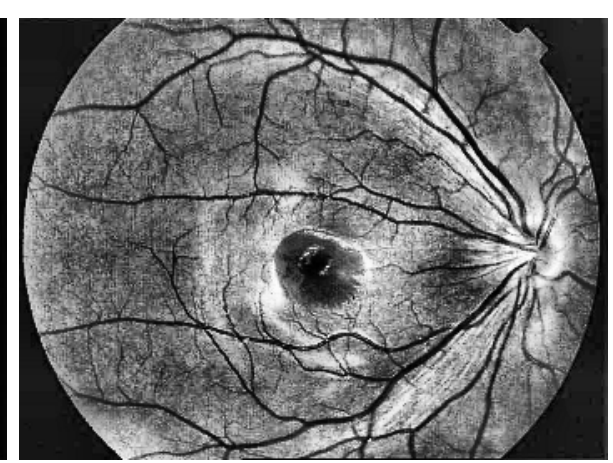

Fig. 2. Gray scale enhanced image 


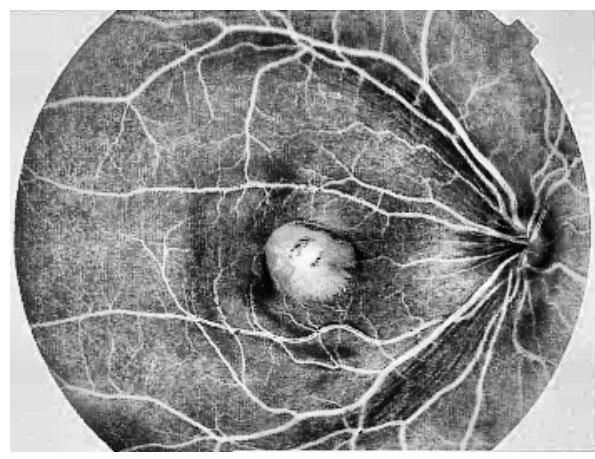

Fig. 3. Inversion to enhance gray scale

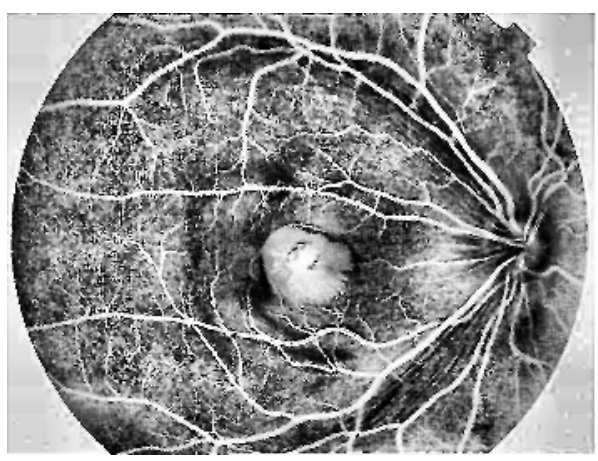

Fig. 4. Filtering

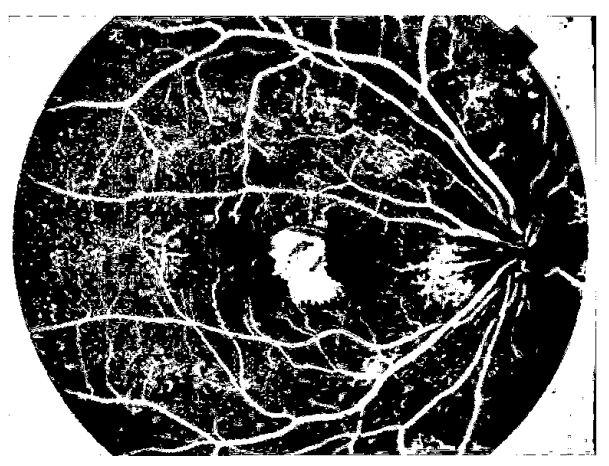

Fig. 5. Binary processing

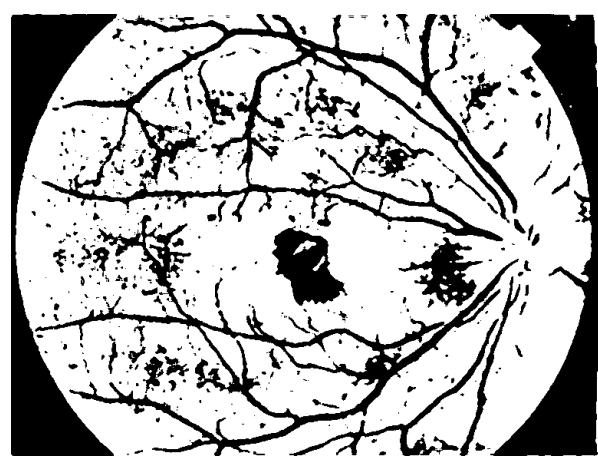

Fig. 7. Fill holes

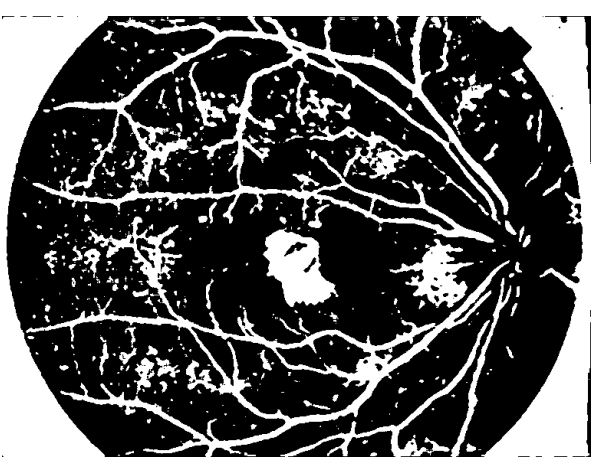

Fig. 6. Median filter

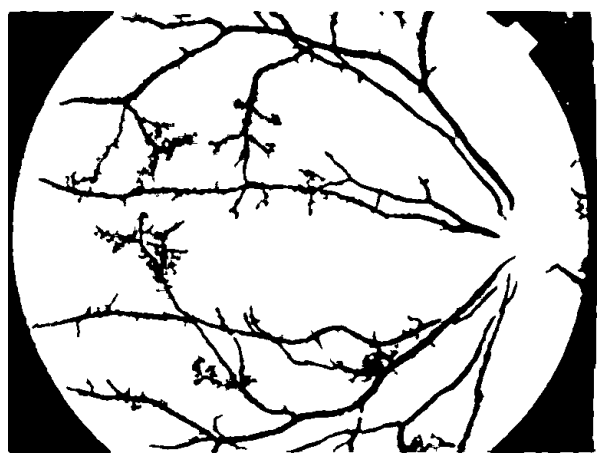

Fig. 8. Erase holes

(2) Filter image by means of un-sharp algorithm(fig.4).

(3) Binary processing. Binary processing deal with all the local maximums over a supposed threshold and input gray scale image as a parameter. In a 

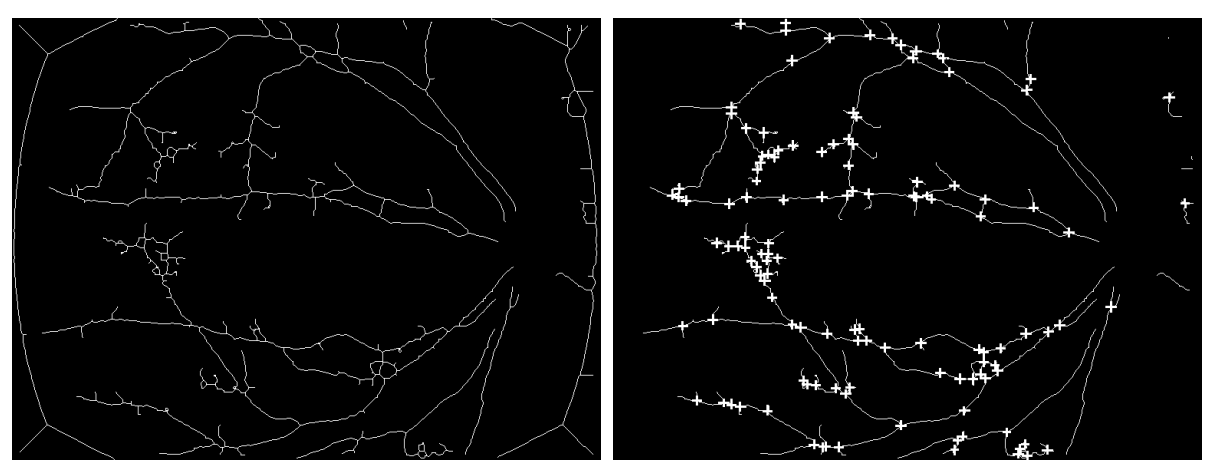

Fig. 9. Extract skeleton

Fig. 10. The cross point of shape of blood vessel

dimorph image, local maximum of output dimorph image is assumed to be 1 , and the remainder 0 , which are used to search out region whose brightness changes most. ( Fig.5). At last, median filter is utilized ( Fig.6).

(4) Fill holes. Holes are those dark regions surrounded by whiter setting. Exchange o with 1 in the binary image (Fig.7) and erase holes (Fig.8).

(5) Extract skeleton on the basis of erosion( Fig.9). Cut edge disturbance and find out the embranchment point of shape of blood vessel. The "+" mark the embranchment point and cross point of shape of blood vessel(Fig.10).

\section{Matching Algorithm for Blood Vessel of Ocular Fundus}

In a bid to transform a given feature point of input image of blood vessel to a corresponding position in the template image of blood vessel, the corresponding transformation factor should be known. The algorithm in this paper deals with image of blood vessel with the same differentiation. In ideal condition, the zoom factor is $\mathrm{Z}$. Assume a given point in the input point set $\mathrm{P}$ is $p_{i}\left(x_{p i}, y_{p i}, \theta_{p i}, 1\right)$, which is transformed by the following formula into $p_{i}\left(x_{p i}^{T}, y_{p i}^{T}, \theta_{p i}^{T}, Z_{p i}^{T}\right)$; and assume a given point in the template point set is $q_{j}\left(x_{q j}, y_{q j}, \theta_{q j}, z_{q j}\right)$. If $\left(x_{p i}^{T}, y_{p i}^{T}, \theta_{p i}^{T}=\right.$ $\left(x_{q j}, y_{q j}, \theta_{q j}, z_{q j}\right)$, the transforming factor is that $p_{i}$ is similar to $q_{j}$ on the condition of $(\triangle \mathrm{x}, \triangle \mathrm{y}, \triangle \theta, \triangle \mathrm{z})$. The images of blood vessel are captured using the same facilities and the same distance. So $p_{i}$ is simplified as $\left(x_{p i}, y_{p i}, \theta_{p i}\right)$ and $q_{j}$ as $\left(x_{q j}, y_{q j}, \theta_{q j}\right)$. The zoom factor in the course of transformation is ruled out.

$$
\left|\begin{array}{c}
x_{p_{i}}^{T} \\
Y_{p_{i}}^{T} \\
\theta_{p_{i}}^{T} \\
1
\end{array}\right|=\left|\begin{array}{cccc}
\cos \triangle \theta & -\sin \triangle \theta & 0 & \triangle x \\
\sin \triangle \theta & \cos \triangle \theta & 0 & \triangle y \\
0 & 0 & 1 & \triangle \theta \\
0 & 0 & 0 & 1
\end{array}\right|\left|\begin{array}{c}
x_{p_{i}} \\
Y_{p_{i}} \\
\theta_{p_{i}} \\
1
\end{array}\right|
$$

Where $\triangle \mathrm{x}$ and $\triangle \mathrm{y}$ are translation factors in $\mathrm{x}$ direction and $\mathrm{y}$ direction respectively, $\triangle \theta$ is rotation factor, and $\triangle \mathrm{Z}=1$ is zoom factor. The first three factors need to be determined in order to identify the two images of blood vessel precisely. 


\subsection{Determination of Reference Point and Calculation of Transformation Factor}

In the process of the point of blood vessel matching, the calculation of matching reference point is of a great importance. Clustering method is utilized to get a precise matching reference point and a group of transformation parameters. Though this method can lead to a quite precise matching reference point, it involves excessive computation. Two images of blood vessel can be transformed in accordance with the obtained transforming parameter, which leads to a further examination of similarity between two triangles.

(1) Calculate respectively corresponding side-length to vertex $p_{i}$ and $q_{j},\left|p_{1} P_{2}\right|$ and $\left|q_{1} q_{2}\right|$.

(2) If ||$p_{1} P_{2}|-| q_{1} q_{2}||>D_{1}$, one corresponding side of the two triangles isn't of the same length, then the two triangles aren't congruent. The examination ends. Re-choose vertices $p_{i}$ and $q_{j}$ and two nearest feature points $\left(p_{1}, p_{2}\right)$ and $\left(q_{1}, q_{2}\right)$. Return to the first step.

(3) Otherwise, calculate respectively the distance form $p_{i}$ to $p_{1}, q_{j}$ to $p_{1}$ and from $p_{2}$ to $q_{1}, p_{2}$ to $q_{2}:\left|p_{i} p_{1}\right|,\left|p_{i} p_{2}\right|$ and $\left|q_{j} q_{1}\right|,\left|q_{j} q_{2}\right|$. If ||$p_{i} p_{1}|-| q_{j} q_{1}|| \leq D_{2}$ and ||$p_{i} p_{2}|-| q_{j} q_{2}|| \leq D_{2}$,or ||$p_{i} p_{2}|-| q_{j} q_{1}|| \leq D_{2}$ and ||$p_{i} p_{1}|-| q_{j} q_{2}|| \leq D_{2}$, it's proved the three sides of the two are of similar length and the two triangles are almost congruent. Otherwise Re-choose vertices $p_{i}$ and $q_{j}$ and two nearest feature points $\left(p_{1}, p_{2}\right)$ and $\left(q_{1}, q_{2}\right)$. Return to the first step.

(4) According to corresponding vertex of the two triangles, calculate orientation disparity between possibly matching feature points, $\triangle \theta_{p_{1} q_{j}}, \triangle \theta_{p_{1} q_{1}}, \triangle \theta_{p_{2} q_{2}}$. The formula is.

$$
\triangle Q_{p q}=\left\{\begin{array}{c}
\theta_{p}-\theta_{q}, \text { if }\left(\theta_{p}-\theta_{q} \geq 0\right) \\
\operatorname{theta}_{p}-\theta_{q}+180, \text { if }\left(\theta_{p}-\theta_{q}<0\right)
\end{array}\right.
$$

If angle disparity between corresponding vertices is similar i.e. $\triangle \theta_{p_{1} q_{j}} \approx$ $\triangle \theta_{p_{1} q_{1}} \approx \triangle \theta_{p_{2} q_{2}}$, the angle between the two feature points $\left(p_{i}, p_{1}, p_{2}\right)$ and $\left(q_{j}, q_{1}, q_{2}\right)$ is supposed to satisfy a rotation relationship. The formula of this rotation is.

$$
\triangle \theta=\frac{1}{3}\left(\theta_{p_{i} q_{j}}+\theta_{p_{1} q_{1}}+\theta_{p_{2} q_{2}}\right)
$$

Otherwise no matching is formed between the sets. Re-choose vertices $p_{i}$ and $q_{j}$ and two nearest feature points $\left(p_{1}, p_{2}\right)$ and $\left(q_{1}, q_{2}\right)$. Return to the first step.

(5) Choose $\left(p_{i}, q_{j}\right)$ as a transforming circle for the rotation and then rotate $\left(q_{j}, q_{1}, q_{2}\right)$. The consequent point is . Calculate spatial disparity in $\mathrm{x}$ direction and y direction respectively which are $\left(\triangle x_{p_{i} q_{j}}, \triangle y_{p_{i} q_{j}}\right),\left(\triangle x_{p_{1} q_{1}}, \triangle y_{p_{1} q_{1}}\right),\left(\triangle x_{p_{2} q_{2}}\right.$, $\left.\triangle y_{p_{2} q_{2}}\right)$. The formula is.

$$
\begin{aligned}
& \Delta x_{p q}=x_{p}-y_{q} \\
& \Delta y_{p q}=y_{p}-y_{q}
\end{aligned}
$$

Now if $\left.\left.\left.\triangle x_{p_{i} q_{j}}\right) \approx \Delta x_{p_{1} q_{1}}\right) \approx \Delta x_{p_{2} q_{2}}\right)$ and $\left.\left.\triangle y_{p_{i} q_{j}}\right) \approx \Delta y_{p_{1} q_{1}}\right) \approx \Delta y_{p_{2} q_{2}}$ ), the two feature point subsets $\left(p_{i}, p_{1}, p_{2}\right)$ and $\left(q_{j}, q_{1}, q_{2}\right)$ meet a kind of transformation 
relationship in $\mathrm{x}, \mathrm{y}$ direction. The two subset match whose rotation, translation and transformation factor are $(\triangle \theta, \triangle x, \triangle y)$ respectively, and

$$
\begin{aligned}
& \triangle x=\frac{1}{3}\left(x_{p_{i} q_{j}}+x_{p_{1} q_{1}}+x_{p_{2} q_{2}}\right) \\
& \triangle y=\frac{1}{3}\left(y_{p_{i} q_{j}}+y_{p_{1} q_{1}}+y_{p_{2} q_{2}}\right)
\end{aligned}
$$

According to the obtained reference point $\left(p_{i}, q_{j}\right)$ and transformation factor $(\triangle \theta$, $\triangle x, \triangle y)$, the skeleton ban of blood vessel be judged whether the same or not.

\section{Experimentation}

In order to identify that the embranchment point of skeleton shape of blood vessel can serve as biometric feature for fundus image of blood vessel, a TRC-50/50VT fundus camera produced form Japanese Topcon company, and fundus images of blood vessel of 2000 people are collected as experimental database where each people has ten images taken at different time. To obtain False Non Match Race, or False Rejection Rate, a matching algorithm is made between every fundus image of blood vessel $T_{i j}$ and its other sample fundus images of blood vessel $F_{i k}(0 \leq j \leq k \leq 9)$ and then the total matching times should be $((10 \mathrm{x} 9) / 2) \mathrm{x}$ $2000=360000$. To obtain False Match Rate, or False Acceptance Rate, a matching algorithm is made between the first sample template $T_{i 0}$ of every fundus blood vessel in the database and the first image $F_{i 0}$ of other fundus blood vessels in the same database. Calculate the ultimate matching result between fundus blood vessel images. The total matching times should be $(2000 x 199) / 2=199000$.

Extracting features from two fundus images of blood vessel follows several steps. At first, according to gray scale image, fix the brightest region of the window, The region's center of the optical disk acts as the origin. Then starting with the horizontal direction; search out the first point of branch of the three vector curves of blood vessel skeleton. This point of branch is regarded as feature point. And then calculate the matching reference point of the two fundus images of blood vessel. The results of the cross-comparison experiment carried out on the 2000 fundus images of blood vessel are: zero false recognition, 25 false rejection and 0.0125 recognition rejection. In this paper, similarity between different fundus skeleton of blood vessel is measured, where four units are adopted as threshold box: 0.65 of fundus skeletons of blood vessel of different people overlap less than $0.292 ; 0.886$ overlap less than $0.51 ; 0.973$ overlap less than 0.73 and 0.9995 overlap less than 90 .

Everyone has contrasting ocular fundus of blood vessel skeleton, which remains constant for long; The diameter of fundus blood vessel does change and capillary vessels do increase but they have no effect on fundus skeleton feature of blood vessel. The feature of embranchment point of blood vessel for ocular fundus is universal, unique and stable but due to certain difficulty as to feature extraction, it hasn't been paid enough attention. At present, with continuous progress in extracting technology, the identification of blood vessel for ocular fundus tends to become an effective method of recognition. 


\section{References}

1. Ulupinar F, Medioni G: Refining edges detected by LoG operator [J].Comput Vis Graph and Image Process, 51:275 298, 1990.

2. Canny J: Acomputational approach to edge detection [J]. IEEE Trans, 8:679 698, PAMI,1986.

3. Peng J, Rusch Ph: Morphological filters and edge detection application to medical imaging [J]. Annual International Conference of the IEEE Engineering In Medicine and Biology Society, 13 (1): 0251 0252, 1991.

4. Huang C C, Li C C, Fan N, et al: A fast morphological filter for enhancement of angiographic images $[\mathrm{J}]$. Annual International Conference of the IEEE Engineering in Medicine and Biology Society, 13(1): 0229 0230, 1991.

5. Tascini G, Passerini G, Puliti P, et al: Retina vascular network recognition [J]. Proc SPIE, 1898: 322 329, 1993.

6. Chauduri S, Chatterjee S, Katz N, et al: Detection of blood vessels in retinal images using two-dimensional matched filters [J]. IEEE Trans Med Imaging,8: 263269 , 1989.

7. T-L Ji, Sundareshan M K, Roehrig H: Adaptive image contrast enhancement based on human visual properties [J]. IEEE Trans Med Imaging,13:573 586, 1994.

8. Matsopoulos George K, Mouravliansky Nicolaos A, Delibasis Konstantinos K, et al: Automatic retinal image registration scheme using global optimization techniques [J]. IEEE Trans On Information Technology In Biomedicine, 3(1): 47 60, 1999.

9. Maes F, Collignon A, Vandermeulen D, et al: Multi-modality image registration by maximization of mutual information [J]. IEEE Trans Med Img, 16 (2): $187198,1997$.

10. Zhan Xiao-Si, Ning Xin-Bao, Yin Yi-Long, Chen Yun: An Improved Point Pattern Algorithm for Fingerprint Matching. Journal of Nanjing University, Vol.39, No.4,pp491 498, July, 2003.

11. Qi Y, Tian J, Deng X: Genetic algorithm based fingerprint matching algorithm and its application on automated fingerprint identification system. Journal of Software,11(4),pp488 493, 2000. 